\title{
Intralesional Therapy in Oncology: A New Vision of a Classic Treatment
}

\author{
Manuel Sureda*, Vanesa Escudero-Ortiz, Elena Martínez-Navarro and Joseba Rebollo \\ Plataforma de Oncología, Hospital Quirónsalud Torrevieja. Torrevieja (Alicante), Spain \\ ^Corresponding author: Manuel Sureda, Plataforma de Oncología, Hospital Quirónsalud Torrevieja. Torrevieja (Alicante), Spain
}

Received: April 28, 2021; Accepted: May 09, 2021; Published: May 10, 2021

\begin{abstract}
Intralesional therapy has been used in oncology for over a century. The former empirical observations on local control were followed by trials with administration of different substances and/or destructive treatments with the aim of controlling cancer growth. Chemotherapy, molecular target therapy, radiotherapy and other physical therapies were initially viewed as a strategy that mainly affected tumor cells, but accumulating evidence in recent years indicates that can also affect the immune system and the tumor microenvironment to contribute to tumor regression. In the light of the recent and relevant findings in immunotherapy, a new point of view that integrates the current knowledge in the topic is presented, with the aim of stimulating further research that might help to optimize the use of new drugs, making them affordable, more effective and less toxic.
\end{abstract}

Keywords: Intralesional therapy, Immunotherapy, Cancer

\section{Introduction}

Intralesional therapy has been used in oncology for over a century, since the initial observations of Coley about tumor remissions in patients treated with bacterial extracts [1]. The former empirical observations on local control were followed by trials with different substances and/or destructive treatments that induced a local acute trauma at the tumor site, with the aim of controlling cancer growth [2,3]. Later on, new reports on distant disease remissions after local injection or Spontaneous Tumor Regression (STR) following infectious episodes and/or surgery caused a progressive shift in the paradigm in parallel with the evolution of knowledge in oncology $[4,5]$.

Everson and Cole defined STR as "the partial or complete disappearance of a malignant tumor in the absence of all treatment, or in the presence of therapy which is considered inadequate to exert significant influence on neoplastic disease" [4]. They also stated in 1966, in a key work in the field, "in many of the collected cases it must be acknowledged that the factors or mechanisms responsible for spontaneous regression are obscure or unknown in the light of present knowledge. However, in some of the cases, available knowledge permits one to infer that hormonal influences probably were important. In other cases, the protocols strongly suggest that an immune mechanism was responsible" [4]. According to many reviews describing STR, Renal Cell Carcinoma (RCC) is one of the most frequent types of malignant neoplasm related to this phenomenon. The rate of STR of RCC, around 1\%, is frequently associated with nephrectomy [6]. Many reports of STR have implicated surgery or operative trauma as an element that can increase immunological resistance to tumor growth. A number of cases in whom surgery on the primary tumor or the metastases has led to regression in the remaining tumor mass have been reported. The removal of a portion of the tumor burden presumably allows the host immune system to destroy the remaining tumor [7].

Initially conceived as an isolated entity growing independently of homeostatic mechanisms of the host, current knowledge of cancer biology has uncovered behind the neoplasia a more complex evolutionary process. Hirata and Shai pointed out this evolutionary nature of cancer, with the Tumor Microenvironment (TME), a complex mixture of non-transformed cell types and extracellular matrix, playing a key role in both the development of tumors and their response to therapy [8]. TME is not merely a physical framework but an interactive structure that conditions tumor development in several ways. In addition, tumors are heterogeneous entities, with strong differences between subpopulations inside the tumor, between primary tumor and metastases and between metastases. Part of this heterogeneity is caused by the presence of Cancer Stem Cells (CSC), characterized by particular phenotypic traits that distinguish them from non-CSC tumor cells. The activation of Epithelial to Mesenchymal Transition (EMT) program has been postulated as the main cause of the epigenetic changes observed in non-CSC to CSC transition. EMT can also activate the possibility of metastatic spread [9]. This plasticity makes possible for carcinoma cells to interconvert between multiple alternative states characterized by different degrees of mesenchymal features [9].

The possible contribution of intralesional therapy to the destruction of all the residual carcinoma cells and the subsequent increased cure rate of different solid tumors can be inferred from the fact that most of cells surviving after various types of therapy commonly display signs of EMT activation. To target cancer cells that have activated portions of the EMT programme constitutes one of the promising ways in development to improve the efficiency of commonly used anticancer therapeutic modalities [9]. 
In a work analyzing the application of Chimeric Antigen Receptor $\mathrm{T}$ (CAR-T) cell therapies to solid tumors, Scarfò and Maus described the identification of proper tumor associated antigens, the limited trafficking of adoptively transferred cells to tumor sites and the immunosuppressive effect of TME as the three main circumstances conditioning its efficacy. TME constitutes a physical barrier decreasing the penetration of modified T-cells into the tumor parenchyma and actively upregulates inhibitory signals. As has been previously suggested, a complete and effective antitumoral strategy has to include all the tumor cells in their different biological situations and the TME elements [10].

Chemotherapy, molecular target therapy, radiotherapy and other physical therapies (electrochemotherapy, cryotherapy, radiofrequency ablation, etc.) were initially conceived as a strategy to destroy tumor cells, but accumulating evidence in recent years indicates that cytotoxic drugs also affect the immune system and TME to contribute to tumor regression. Local irradiation of a single tumor site can reduce the size of non-irradiated metastases that are located at a distant site, a phenomenon known as abscopal effect, mediated by the immune system. Neoadjuvant radiochemotherapy may be immunologically more relevant than adjuvant therapies [11]. Several anticancer therapies have "yin-yang" characteristics with respect to the tumor, being able to attack tumor cells but to cause immunosuppression as well, with the response to the treatment depending on the balance between both aspects. It has been postulated that there is no cancer cure without inducing effective antitumor immunity, independently of the therapeutic modalities employed to treat the patient [11].

From an immunological point of view, tumors are classified as "hot" (T cell-infiltrated), "excluded" (inflamed but non-infiltrated), "immunosuppressed" (low infiltration in tumor and margins) and "cold" (non-inflamed) [12]. According to the previous postulates, physical therapies can cause tumor response by direct destruction of tumor cells, induction of immunogenic cell death with the release of danger associated molecular patterns, and tumor associated antigens that are key to initiate an innate immune response, targeting both the treated lesion as well as distinct lesions. These physical therapies can be combined with classical treatment modalities (chemotherapy, radiotherapy) and/or intralesional immunomodulating factors (BCG, IL2, IFNa, oncolytic viruses, etc), with the aim to enhance anti-tumor immune responses ${ }^{2}$ rendering hot an initially described as cold or altered tumor.

Some systemic immunotherapies have a benefit limited to a minority of patients, might cause immune-related adverse events and imply long-lasting treatments with the risk of financial toxicity. Intratumoral immunotherapy can address these issues by providing a better priming of the antitumor immune response, avoiding off-target toxicities and requiring a lower amount of medication per patient [13]. Disruption of physical barriers, local activation of immune effectors, inactivation of immunosuppressant cellular populations (myeloid derived suppressor cells, Tregs, tumor associated macrophages) and counteraction of soluble immunosuppressant factors can be better attained by direct action on the tumor mass, with a more selective and less toxic effect, with the possibility of expanding combinations of complementary agents (probably not feasible by systemic route) and at a much lower cost. The most exciting modality in this field has been the intratumoral administration of oncolytic viruses. Talimogene laherparepvec, the first approved by FDA, is a genetically engineered herpes virus with two genes removed-one that shuts down an individual cell's defenses, and another that helps the virus evade the immune system-and the one that codes for human GM-CSF added. Initial results in patients with previously resistant metastatic melanoma have been encouraging.

In recent years, several other attempts have been made in order to induce local secretion of immunomodulating molecules as IL12 or GM-CSF in the tumor area through genetic engineering of somatic and/or tumor cells, with the aim of obtaining a clinical response. They have paved an exciting way, but at this moment, in general, results have not been conclusive enough and the question if it represents an improvement over direct intralesional administration of the substances is not resolved. In summary, intralesional route in cancer therapy has evolved from an empirical concept to a more defined one, with exciting possibilities strongly related with the development of modern immunotherapy and a future open to new combinations. Further studies to better delineate this field are warranted.

\section{Declarations}

1. Funding: This work has no funding sources.

2. Conflicts of interest/Competing interests: The authors declare no conflicts of interest.

3. Ethics approval: N/A.

4. Consent to participate (include appropriate statements) N/A.

5. Consent for publication (include appropriate statements) N/A.

6. Availability of data and material (data transparency) N/A.

7. Code availability (software application or custom code) N/A.

8. Authors' contributions: MS conceptualization, formal analysis, supervision, writing original draft, writing review \& editing; VEO conceptualization, formal analysis, writing review \& editing; EMN data curation, investigation, visualization, writing review \& editing; JR data curation, investigation, writing review \& editing.

\section{References}

1. Coley WB (1991) The treatment of malignant tumors by repeated inoculations of erysipelas: With a report of ten original cases. Am J Med Sci 105: 487-510. [crossref]

2. Locy H, de Mey S, de Mey W, De Ridder M, Thielemans K, et al. (2018) Immunomodulation of the tumor microenvironment: Turn foe into friend. Front Immunol 9: 2909. [crossref]

3. Aznar MA, Tinari N, Rullán AJ, Sánchez-Paulete AR, Rodriguez-Ruiz ME, et al. (2017) Intratumoral delivery of immunotherapy-act locally, think globally. J Immunol 198: 31-39. [crossref]

4. Everson T, Cole WH (1996) Spontaneous regression of cancer. Philadelphia, Penn: JB Saunders \& Co.

5. Sureda M, Subirá ML, Martín Algarra S, Prieto Valtueña J, Sangro B (1990) Spontaneous tumor regression. Report of 2 cases. Med Clin (Barc) 95: 306-308. [crossref] 
6. Snow RM, Schellhammer PF (1982) Spontaneous regression of metastatic renal cell carcinoma. Urology 20: 177-181.

7. Cole WH (1974) Spontaneous regression of cancer: The metabolic triumph of the host? Ann NY Acad Sci 230: 111-141. [crossref]

8. Hirata E, Sahai E (2017) Tumor microenvironment and differential responses to therapy. Cold Spring Harb Perspect Med 7: 026781. [crossref]

9. Shibue T, Weinberg RA (2017) EMT, CSCs, and drug resistance: The mechanistic link and clinical implications. Nat Rev Clin Oncol 14: 611-629. [crossref]
10. Scarfò I, Maus M (2017) Current approaches to increase CAR T cell potency in solid tumors: Targeting the tumor microenvironment. J Immunother Cancer 5: 28. [crossref]

11. Zitvogel L, Apetoh L, Ghiringhelli F, Kroemer G (2008) Immunological aspects of cancer chemotherapy. Nat Rev Immunol 8: 59-73. [crossref]

12. Galon J, Bruni D (2019) Approaches to treat immune hot, altered and cold tumours with combination immunotherapies. Nat Rev Drug Discov 18: 197-218. [crossref]

13. Marabelle A, Tselikas L, de Baere T, Houot R (2017) Intratumoral immunotherapy: Using the tumor as the remedy. Ann Oncol 28: 33-43. [crossref]

\section{Citation:}

Sureda M, Escudero-Ortiz V, Navarro EM, Rebollo J (2021) Intralesional Therapy in Oncology: A New Vision of a Classic Treatment. Cancer Stud Ther J Volume 6(2): 1-3. 